\title{
FIXED POINT RESULTS FOR HARDY-ROGERS TYPE CONTRACTIONS WITH RESPECT TO A $C$-DISTANCE IN GRAPHICAL CONE METRIC SPACES
}

\begin{abstract}
The aim of this paper is to prove some existence and uniqueness results of the fixed points for Hardy-Rogers type contraction in cone metric spaces associated with a $c$-distance and endowed with a graph. These results prepare a more general statement, since we apply the condition of orbitally $G$-continuity of mapping instead of the condition of continuity, and consider cone metric spaces endowed with a graph instead of cone metric spaces.
\end{abstract}

Key words: c-distance, cone metric spaces, fixed point, orbitally $G$-continuous, connected graph

2010 Mathematical Subject Classification: 46A19, 47H10, 05C20

1. Introduction and preliminaries. In 1996, Kada et al. [11] defined the concept of $w$-distance in metric spaces and proved some fixed point theorems with respect to this distance. On the other hand, in 1997, Zabrejko [18] developed a fixed point theory in abstract metric spaces and $K$-normed spaces. Later, Huang and Zhang [8] reintroduced the concept of the cone metric space by replacing the set of real numbers by an ordered Banach space. In 2011, Cho et al. [3] defined a cone version of the $w$-distance (where it is called $c$-distance) and obtained some fixed point theorems under a $c$-distance in ordered cone metric spaces. For more results, see the papers [7] by Huang et al. and [15], [16] by Rahimi and Soleimani Rad. Further, in 2008, Jachymski [9] equipped the underlying metric space with a directed graph and formulated the Banach contraction in the graph language. After that, some authors extended the fixed point theory in the graph language in [1], [12], [14], see also references therein. Very recently, Fallahi et al. [4], [5] studied the existence of the

(C) Petrozavodsk State University, 2020 
fixed points for various contractive mappings with respect to a $c$-distance in cone metric spaces endowed with a graph.

In this paper, we consider a $c$-distance in cone metric spaces with a directed graph and obtain some fixed point theorems of Hardy-Rogers type contraction with respect to this distance. We start by reviewing a few basic definitions and notions, which are frequently applied.

Definition 1. [8] Let $E$ be a real Banach space with the zero element $\theta$. $A$ proper nonempty and closed subset $P$ of $E$ is called a cone if $P+P \subset P$, $\lambda P \subset P$ for $\lambda \geqslant 0$ and $P \cap(-P)=\{\theta\}$.

Given a cone $P \subset E$, Huang and Zhang [8] applied a partial ordering $\preceq$ with respect to $P$ by $x \preceq y$ if and only if $y-x \in P$. We write $x \prec y$ if $x \preceq y$ and $x \neq y$. Moreover, we denote $x \ll y$ if and only if $y-x \in \operatorname{int} P$, where int $P$ is the interior of $P$. If int $P \neq \theta$, then the cone $P$ is named solid. The cone $P$ is normal if there is a number $k>0$ such that for all $x, y \in E$, where $\theta \preceq x \preceq y$, we have $\|x\| \leqslant k\|y\|$.

Definition 2. [8] Let $X$ be a nonempty set. Suppose that the mapping $d: X \times X \rightarrow P$ satisfies

(d1) $\theta \preceq d(x, y)$ for all $x, y \in X$ and $d(x, y)=\theta$ if and only if $x=y$;

(d2) $d(x, y)=d(y, x)$ for all $x, y \in X$;

(d3) $d(x, z) \preceq d(x, y)+d(y, z)$ for all $x, y, z \in X$.

Then $d$ is named a cone metric on $X$ and $(X, d)$ is called a cone metric space.

For other notions and concepts, such as Cauchy sequences, convergence, completeness, and continuity in cone metric spaces, we refer to [8]. We shall also make use of the following property when the cone $P$ is nonnormal:

(*) Let $u \preceq \lambda u$ with $u \in P$ and $0<\lambda<1$. Then $u=\theta$.

Definition 3. [3], [17] Let $(X, d)$ be a cone metric space. A function $q: X \times X \rightarrow E$ is called a c-distance on $X$ if the following holds:

$\left(q_{1}\right) \theta \preceq q(x, y)$ for all $x, y \in X$;

$\left(q_{2}\right) q(x, z) \preceq q(x, y)+q(y, z)$ for all $x, y, z \in X$;

$\left(q_{3}\right)$ for all $n \geqslant 1$ and $x \in X$, if $q\left(x, y_{n}\right) \preceq u$ for some $u=u_{x}$, then $q(x, y) \preceq u$ whenever $\left\{y_{n}\right\}$ is a sequence in $X$ converging to a point $y \in X$; 
$\left(q_{4}\right)$ for all $c \in E$ with $c \in \operatorname{int} P$, there exists $e \in E$ with $e \in \operatorname{int} P$ such that $q(z, y) \ll e$ and $q(z, x) \ll e$ imply $d(x, y) \ll c$.

Each $w$-distance is a $c$-distance in a cone metric space with $E=\mathbb{R}$ and $P=[0, \infty)$. But the converse does not hold. Thus, the $c$-distance is a generalization of the $w$-distance. Moreover, for a $c$-distance $q, q(a, b)=\theta$ is not necessarily equivalent to $a=b$ and $q(a, b)=q(b, a)$ does not necessarily hold for all $a, b \in X$.

Lemma 1. [3], [7] Let $(X, d)$ be a cone metric space, $q$ be a $c$-distance on $X,\left\{a_{n}\right\}$ be a sequence in $X$, and $\left\{\gamma_{n}\right\}$ and $\left\{\delta_{n}\right\}$ be two convergent sequences to $\theta$ in $P$. For any $a, b, c \in X$, the following properties hold:

(i) let $q\left(a_{n}, b\right) \preceq \gamma_{n}$ and $q\left(a_{n}, c\right) \preceq \delta_{n}$ for all $n \in \mathbb{N}$. Then $b=c$. Also, if $q(a, b)=\theta$ and $q(a, c)=\theta$, then $b=c$;

(ii) let $q\left(a_{n}, a_{m}\right) \preceq \gamma_{n}$ for all $m, n \in \mathbb{N}$ with $m>n$. Then $\left\{a_{n}\right\}$ is a Cauchy sequence in $X$.

Let $(X, d)$ be a cone metric space and $G$ be a directed graph without parallel edges and with the vertex set $V(G)=X$ and the edge set $E(G)$ that contains all loops. Then the graph $G$ can be written as the ordered pair $(V(G), E(G))$ and $(X, d)$ is the named cone metric space endowed with the graph $G$. Also, The graph $G$ is connected if there exists a path in $G$ between every two vertices of $G$. For more details on graphs, see [2]. In the sequel, let $(X, d)$ be a cone metric space endowed with a graph $G$ with $V(G)=X$ and $\Delta(X) \subseteq E(G)$, where $\Delta(X)=\{(x, x) \in X \times X$ : $x \in X\}$, Fix $(T)$ be the set of all fixed points of a self-map $T$ on $X$ and $X_{T}=\{x \in X:(x, T x) \in E(G)\}$.

From the idea of Jachymski [9] and Petruşel and Rus [13], Fallahi et al. defined Picard operators in cone metric spaces and orbitally $G$-continuous mappings on $X$ as follows.

Definition 4. [4], [5] Let $(X, d)$ be a cone metric space. A self-map $T$ on $X$ is called a Picard operator if $T$ has a unique fixed point $x_{*}$ in $X$ and $T^{n} x \rightarrow x_{*}$ for all $x \in X$.

Definition 5. [4], [5] Let $(X, d)$ be a cone metric space endowed with a graph $G$. A mapping $T: X \rightarrow X$ is called orbitally $G$-continuous on $X$ if for all $x, y \in X$ and all sequences $\left\{b_{n}\right\}$ of positive integers with $\left(T^{b_{n}} x, T^{b_{n+1}} x\right) \in E(G)$ for all $n \geqslant 1$, the convergence $T^{b_{n}} x \rightarrow y$ implies $T\left(T^{b_{n}} x\right) \rightarrow T y$. 
Note that a continuous mapping on a cone metric space is orbitally $G$-continuous for all graphs $G$, but the converse is not generally true.

2. Main results. The following theorem is the principal result of this paper; it uses Hardy-Rogers contraction [6].

Theorem 1. Let $(X, d)$ be a complete cone metric space endowed with a graph $G, q$ be a $c$-distance, and $T: X \rightarrow X$ be an orbitally $G$-continuous mapping that preserves the edges of $G$; that is, $(x, y) \in E(G)$ implies $(T x, T y) \in E(G)$ for all $x, y \in X$. Suppose that there exist mappings $\nu_{i}: X \rightarrow[0,1)$ with $\nu_{i}(T x) \leqslant \nu_{i}(x)$ for all $x \in X$ and for $i=1,2, \ldots, 5$, such that

$$
\begin{aligned}
q(T x, T y) \preceq & \nu_{1}(x) q(x, y)+\nu_{2}(x) q(x, T x)+\nu_{3}(x) q(y, T y)+ \\
& +\nu_{4}(x) q(x, T y)+\nu_{5}(x) q(y, T x) \\
q(T y, T x) \preceq & \nu_{1}(x) q(y, x)+\nu_{2}(x) q(T x, x)+\nu_{3}(x) q(T y, y)+ \\
& +\nu_{4}(x) q(T y, x)+\nu_{5}(x) q(T x, y)
\end{aligned}
$$

for all $x, y \in X$ with $(x, y) \in E(G)$, where

$$
\left(\nu_{1}+\nu_{2}+\nu_{3}+2 \nu_{4}+2 \nu_{5}\right)(x)<1 .
$$

Then $X_{T} \neq \emptyset$ if and only if $T$ has a fixed point. Further, if $T z=z$, then $q(z, z)=\theta$. Moreover, if the subgraph of $G$ with the vertex set Fix $(T)$ is connected, then the restriction of $T$ to $X_{T}$ is a Picard operator.

Proof. Because Fix $(T) \subseteq X_{T}$, if $T$ has a fixed point, then $X_{T}$ is nonempty. Conversely, let $x_{0} \in X_{T}$. Since $T$ preserves the edges of $G$, then $\left(x_{n}, x_{n+1}\right) \in$ $\in E(G)$ for all $n \in \mathbb{N}$, where $x_{n}=T x_{n-1}=T^{n} x_{0}$. Now, by considering $x=x_{n}$ and $y=x_{n-1}$ in (1) and since $\left(x_{n-1}, x_{n}\right) \in E(G)$, we have

$$
\begin{aligned}
& q\left(x_{n+1}, x_{n}\right)=q\left(T x_{n}, T x_{n-1}\right) \preceq \\
& \preceq \nu_{1}\left(x_{n}\right) q\left(x_{n}, x_{n-1}\right)+\nu_{2}\left(x_{n}\right) q\left(x_{n}, T x_{n}\right)+\nu_{3}\left(x_{n}\right) q\left(x_{n-1}, T x_{n-1}\right)+ \\
& +\nu_{4}\left(x_{n}\right) q\left(x_{n}, T x_{n-1}\right)+\nu_{5}\left(x_{n}\right) q\left(x_{n-1}, T x_{n}\right) \preceq \\
& \quad \vdots \\
& \preceq \nu_{1}\left(x_{0}\right) q\left(x_{n}, x_{n-1}\right)+\left(\nu_{3}+\nu_{5}\right)\left(x_{0}\right) q\left(x_{n-1}, x_{n}\right)+\nu_{4}\left(x_{0}\right) q\left(x_{n+1}, x_{n}\right) \\
& +\left(\nu_{2}+\nu_{4}+\nu_{5}\right)\left(x_{0}\right) q\left(x_{n}, x_{n+1}\right)
\end{aligned}
$$

Similarly, by considering $x=x_{n}$ and $y=x_{n-1}$ in (2), we have

$$
q\left(x_{n}, x_{n+1}\right) \preceq \nu_{1}\left(x_{0}\right) q\left(x_{n-1}, x_{n}\right)+\left(\nu_{3}+\nu_{5}\right)\left(x_{0}\right) q\left(x_{n}, x_{n-1}\right)+
$$




$$
+\nu_{4}\left(x_{0}\right) q\left(x_{n}, x_{n+1}\right)+\left(\nu_{2}+\nu_{4}+\nu_{5}\right)\left(x_{0}\right) q\left(x_{n+1}, x_{n}\right) .
$$

Adding up (4) and (5), we obtain

$$
\begin{aligned}
q\left(x_{n+1}, x_{n}\right)+q\left(x_{n}, x_{n+1}\right) & \preceq\left(\nu_{1}+\nu_{3}+\nu_{5}\right)\left(x_{0}\right)\left[q\left(x_{n}, x_{n-1}\right)+q\left(x_{n-1}, x_{n}\right)\right] \\
& +\left(\nu_{2}+2 \nu_{4}+\nu_{5}\right)\left(x_{0}\right)\left[q\left(x_{n+1}, x_{n}\right)+q\left(x_{n}, x_{n+1}\right)\right] .
\end{aligned}
$$

Put $\mu_{n}=q\left(x_{n+1}, x_{n}\right)+q\left(x_{n}, x_{n+1}\right)$. Then

$$
\mu_{n} \preceq\left(\nu_{1}+\nu_{3}+\nu_{5}\right)\left(x_{0}\right)\left(\mu_{n-1}\right)+\left(\nu_{2}+2 \nu_{4}+\nu_{5}\right)\left(x_{0}\right) \mu_{n},
$$

which implies $\mu_{n} \preceq k \mu_{n-1}$ for all $n \in \mathbb{N}$, where

$$
0 \leqslant k=\frac{\left(\nu_{1}+\nu_{3}+\nu_{5}\right)\left(x_{0}\right)}{1-\left(\nu_{2}+2 \nu_{4}+\nu_{5}\right)\left(x_{0}\right)}<1
$$

by $(3)$ and since $\left(\nu_{1}+\nu_{3}+\nu_{5}\right)\left(x_{0}\right) \geqslant 0$. By repeating this procedure, we have $\mu_{n} \preceq k^{n} \mu_{0}$ for all $n \in \mathbb{N}$. Hence,

$$
q\left(x_{n}, x_{n+1}\right) \preceq \mu_{n} \preceq k^{n}\left[q\left(x_{1}, x_{0}\right)+q\left(x_{0}, x_{1}\right)\right] .
$$

Now, let $m>n$. It follows from $\left(q_{2}\right),(6)$ and (7) that

$$
q\left(x_{n}, x_{m}\right) \preceq \frac{k^{n}}{1-k}\left[q\left(x_{1}, x_{0}\right)+q\left(x_{0}, x_{1}\right)\right] .
$$

Since $\frac{k^{n}}{1-k}\left[q\left(x_{1}, x_{0}\right)+q\left(x_{0}, x_{1}\right)\right]$ is a convergent to $\theta$ sequence, Lemma 1 (ii) implies that $\left\{x_{n}\right\}$ is a Cauchy sequence. Since $X$ is complete, there exists point $z \in X$ such that $x_{n}=T^{n} x_{0} \rightarrow z$ as $n \rightarrow \infty$. Now, we prove that $z$ is a fixed point for $T$. From $x_{0} \in X_{T}$ we have $\left(T^{n} x_{0}, T^{n+1} x_{0}\right) \in E(G)$ for all $n \geqslant 0$. Thus, by the orbital $G$-continuity of $T$, we have $T^{n+1} x_{0} \rightarrow T z$. Since the limit of a sequence is unique, we conclude $T z=z$ and $z$ is a fixed point of the mapping $T$. Also, let $T z=z$ for $z \in X$. It follows from (1) that $q(z, z) \preceq\left(\nu_{1}+\nu_{2}+\nu_{3}+\nu_{4}+\nu_{5}\right)(z) q(z, z)$. Since $\sum_{i=1}^{5} \nu_{i}(z)<$ $<\left(\nu_{1}+\nu_{2}+\nu_{3}+2 \nu_{4}+2 \nu_{5}\right)(z)<1$, then $q(z, z)=\theta$ by $(*)$.

Next, suppose that the subgraph of $G$ with the vertex set $\operatorname{Fix}(T)$ is connected and $z_{*} \in X$ is a fixed point of $T$. Then there exists a path $\left(x_{i}\right)_{i=0}^{N}$ in $G$ from $z$ to $z_{*}$, such that $x_{1}, \ldots, x_{N-1} \in \operatorname{Fix}(T)$ by $x_{0}=z, x_{N}=z_{*}$ and $\left(x_{i-1}, x_{i}\right) \in E(G)$ for $i=1, \ldots, N$. Since $q\left(x_{i-1}, x_{i-1}\right)=q\left(x_{i}, x_{i}\right)=\theta$ for each $i=1,2, \ldots, N$ and by applying (1) and (2), we get

$$
q\left(x_{i}, x_{i-1}\right) \preceq\left(\nu_{1}+\nu_{4}\right)\left(x_{i}\right) q\left(x_{i}, x_{i-1}\right)+\nu_{5}\left(x_{i}\right) q\left(x_{i-1}, x_{i}\right),
$$




$$
q\left(x_{i-1}, x_{i}\right) \preceq\left(\nu_{1}+\nu_{4}\right)\left(x_{i}\right) q\left(x_{i-1}, x_{i}\right)+\nu_{5}\left(x_{i}\right) q\left(x_{i}, x_{i-1}\right) .
$$

Now, adding up (8) and (9), we have

$$
q\left(x_{i}, x_{i-1}\right)+q\left(x_{i-1}, x_{i}\right) \preceq\left(\nu_{1}+\nu_{4}+\nu_{5}\right)\left(x_{i}\right)\left[q\left(x_{i}, x_{i-1}\right)+q\left(x_{i-1}, x_{i}\right)\right],
$$

which implies that $q\left(x_{i}, x_{i-1}\right)+q\left(x_{i-1}, x_{i}\right)=\theta$ by $(3)$ and $\left(\nu_{1}+\nu_{4}+\nu_{5}\right)\left(x_{i}\right) \preceq$ $\preceq\left(\nu_{1}+\nu_{2}+\nu_{3}+2 \nu_{4}+2 \nu_{5}\right)\left(x_{i}\right)$. Hence, $q\left(x_{i}, x_{i-1}\right)=q\left(x_{i-1}, x_{i}\right)=\theta$. Thus, by Lemma 1 (i) and since $q\left(x_{i}, x_{i}\right)=\theta$ and $q\left(x_{i}, x_{i-1}\right)=\theta$, we have $x_{i}=x_{i-1}$ for $i=1,2, \ldots, N$; that is, $z=x_{0}=x_{1}=\cdots=x_{N-1}=x_{N}=z_{*}$. Therefore, the fixed point of $T$ is unique and the restriction of $T$ to $X_{T}$ is a Picard operator. This completes the proof.

Example. Let $X=[0,1], E=C_{\mathbb{R}}^{1}[0,1]$ with the norm $\|\psi\|=\|\psi\|_{\infty}+\left\|\psi^{\prime}\right\|_{\infty}$, $P=\{\psi \in E: \psi(t) \geqslant 0$ on $[0,1]\}$ be a non-normal cone. Consider the mapping $d: X \times X \rightarrow E$ introduced by $d(x, y)=|x-y| \cdot \psi(t)$ for all $x, y \in X$, where $\psi(t)=e^{t} \in P \subset E$ with $t \in[0,1]$. Then $(X, d)$ is a cone metric space with a solid cone. Define the mapping $q: X \times X \rightarrow E$ by $q(x, y)(t)=y \cdot e^{t}$ for all $x, y \in X$, where $t \in[0,1]$. Then $q$ is a $c$ distance. Define $T: X \rightarrow X$ by $T(x)=\frac{x^{2}}{4}$, if $x \neq \frac{1}{2}$, and $T x=0$, if $x=\frac{1}{2}$. Clearly, $T$ is not continuous at $x=\frac{1}{2}$, and, so, on the whole $X$. Suppose that $X$ is endowed with a graph $G=(V(G), E(G))$, where $V(G)=X$ and $E(G)=\{(x, x): x \in X\} \cup\left\{\left(0, \frac{1}{2}\right),\left(\frac{1}{2}, 0\right)\right\}$. Now, $T$ is orbitally $G$-continuous on $X$. Consider mappings $\nu_{1}(x)=\frac{x+1}{4}, \nu_{2}(x)=\frac{x}{4}$ and $\nu_{3}(x)=\nu_{4}(x)=\nu_{5}(x)=0$ for all $x \in X$. Then

(i) if $x \neq \frac{1}{2}$, then $\nu_{1}(T x)=\nu_{1}\left(\frac{x^{2}}{4}\right)=\frac{\frac{x^{2}}{4}+1}{4} \leqslant \frac{x+1}{4}=\nu_{1}(x)$ and if $x=\frac{1}{2}$, then $\nu_{1}\left(T \frac{1}{2}\right)=\frac{1}{4} \leqslant \frac{3}{8}=\nu_{1}\left(\frac{1}{2}\right)$;

(ii) if $x \neq \frac{1}{2}$, then $\nu_{2}(T x)=\nu_{2}\left(\frac{x^{2}}{4}\right)=\frac{x^{2}}{16} \leqslant \frac{x}{4}=\nu_{2}(x)$ and if $x=\frac{1}{2}$, then $\nu_{2}\left(T \frac{1}{2}\right)=0 \leqslant \frac{1}{8}=\nu_{2}\left(\frac{1}{2}\right)$

(iii) $\nu_{i}(T x) \leqslant \nu_{i}(x)$ for all $x \in X$ and $i=3,4,5$;

(iv) $\left(\nu_{1}+\nu_{2}+\nu_{3}+2 \nu_{4}+2 \nu_{5}\right)(x)=\frac{x+1}{4}+\frac{x}{4}<1$ for all $x \in X$;

(v) let $x \in X$ with $(x, x) \in E(G)$. Then, in two cases $x=\frac{1}{2}$ and $x \neq \frac{1}{2}$, both relations (1) and (2) are true;

(vi) since $(0, T 0)=(0,0) \in E(G)$, we have $X_{T} \neq \emptyset$.

Thus, all the conditions of Theorem 1 are true. Clearly, $T$ has a unique fixed point $x=0 \in[0,1]$ and $q(0,0)=0$. 
Now, several consequences of our main result follow for particular choices of the graph $G$. Firstly, consider a cone metric space $(X, d)$ endowed with the complete graph $G_{0}$, whose vertex set coincides with $X$; that is, $V\left(G_{0}\right)=X$ and $E\left(G_{0}\right)=X \times X$. Let $G=G_{0}$ in Theorem 1 . Then it is clear that the set $X_{T}$ related to any self-map $T$ on $X$ coincides with the whole set $X$. Thus, we have the following corollary.

Corollary 1. Let $(X, d)$ be a complete cone metric space endowed with the graph $G_{0}, q$ be a $c$-distance and $T: X \rightarrow X$ be a orbitally $G_{0^{-}}$ continuous mapping. Suppose that there exist mappings $\nu_{i}: X \rightarrow[0,1)$ with $\nu_{i}(T x) \leqslant \nu_{i}(x)$ for all $x \in X$ and for $i=1,2, \ldots, 5$, such that relations (1)-(3) hold for all $x, y \in X$. Then $T$ has a fixed point.

Now, let $(X, \sqsubseteq)$ be a poset (partially ordered set) and $G_{1}$ be the graph with $V\left(G_{1}\right)=X$ and $E\left(G_{1}\right)=\{(x, y) \in X \times X: x \sqsubseteq y\}$. Since $\sqsubseteq$ is reflexive, $E\left(G_{1}\right)$ contains all loops. By putting $G=G_{1}$ in Theorem 1, we obtain the following corollary of our main theorem.

Corollary 2. Let $(X, \sqsubseteq)$ be a poset, $(X, d)$ be a complete cone metric space, $q$ be a $c$-distance, and $T: X \rightarrow X$ be a nondecreasing and orbitally $G_{1}$-continuous mapping on $X$. Suppose that there exist mappings $\nu_{i}$ : $X \rightarrow[0,1)$ with $\nu_{i}(T x) \leqslant \nu_{i}(x)$ for all $x \in X$ and for $i=1,2, \ldots, 5$, such that relations (1)-(3) hold for all $x, y \in X$ with $x \sqsubseteq y$. Then $T$ has a fixed point if and only if there exist $x_{0} \in X$ such that $x_{0} \sqsubseteq T x_{0}$. Further, if $T z=z$, then $q(z, z)=\theta$.

Now, let $X$ be a poset endowed with the graph $G_{2}$ given by $V\left(G_{2}\right)=X$ and $E\left(G_{2}\right)=\{(x, y) \in X \times X: x \sqsubseteq y \vee y \sqsubseteq x\}$; that is, an ordered pair $(x, y) \in X \times X$ is an edge of $G_{2}$ if and only if $x$ and $y$ are comparable elements of $(X, \sqsubseteq)$. Consider $G=G_{2}$ in Theorem 1. Then we have another fixed point corollary as follows.

Corollary 3. Let $(X, \sqsubseteq)$ be a poset, $(X, d)$ be a complete cone metric space, $q$ be a c-distance, and $T: X \rightarrow X$ be a nondecreasing and orbitally $G_{2}$-continuous mapping that maps comparable elements of $X$ onto comparable elements. Suppose that there exist mappings $\nu_{i}: X \rightarrow[0,1)$ with $\nu_{i}(T x) \leqslant \nu_{i}(x)$ for all $x \in X$ and for $i=1,2, \ldots, 5$, such that relations (1) - (3) hold for all comparable $x, y \in X$. Then $T$ has a fixed point on $X$ if and only if there exist $x_{0} \in X$ such that $x_{0}$ and $T x_{0}$ are comparable. Moreover, if $T z=z$, then $q(z, z)=\theta$.

Let $\varepsilon \in \operatorname{int} P$ be fixed. Two elements $x, y \in X$ are said to be $\varepsilon$-closed 
if $d(x, y) \prec \varepsilon$. Consider the $\varepsilon$-graph $G_{3}$ with $V\left(G_{3}\right)=X$ and $E\left(G_{3}\right)=$ $=\{(x, y) \in X \times X: d(x, y) \prec \varepsilon\}$. Note that $E\left(G_{3}\right)$ contains all loops. Set $G=G_{3}$ in Theorem 1. Then we have the following result.

Corollary 4. Let $(X, d)$ be a complete cone metric space endowed with the graph $G_{3}, \varepsilon \in \operatorname{int} P, q$ be a c-distance, and $T: X \rightarrow X$ be a orbitally $G_{3}$-continuous mapping that maps $\varepsilon$-close elements of $X$ onto $\varepsilon$ close elements. Suppose that there exist mappings $\nu_{i}: X \rightarrow[0,1)$ with $\nu_{i}(T x) \leqslant \nu_{i}(x)$ for all $x \in X$ and for $i=1,2, \ldots, 5$, such that relations (1) - (3) hold for all $x, y \in X$ such that $x$ and $y$ are $\varepsilon$-close elements. Then $T$ has a fixed point on $X$ if and only if there exists $x_{0} \in X$ such that $x_{0}$ and $T x_{0}$ are $\varepsilon$-close. Moreover, if $T z=z$, then $q(z, z)=\theta$.

Remark 1. In Theorem 1 and its corollaries, consider $\nu_{i}(x)=\nu_{i}$ for $i=1,2, \ldots, 5$. Then we can obtain the same assertions. Also, for Banachtype fixed point result with respect to a c-distance on cone metric spaces endowed with a graph, we apply the condition $q(T x, T y) \preceq \alpha q(x, y)$ for all $x, y \in X$, where $\alpha \in[0,1)$.

3. Application to a fourth-order differential equation. In this section, the existence of solution of a fourth-order boundary-value problem by applying Green's functions is established as a consequence of Corollary 2. Specially, we study the fourth-order two-point boundary value problem

$$
\left\{\begin{array}{l}
x^{i v}(t)=k(t, x(t)), \quad 0<t<1, \\
x(0)=x^{\prime}(0)=x^{\prime \prime}(1)=x^{\prime \prime \prime}(1)=0,
\end{array}\right.
$$

with $k \in C([0,1] \times \mathbb{R}, \mathbb{R})$. Note that the problem (10) may be equivalently expressed in the integral form: find the solution $x^{*} \in X$ of

$$
x(t)=\int_{0}^{1} G(t, \tau) k(\tau, x(\tau)) d \tau, \quad t \in[0,1],
$$

where the Green function $G(t, \tau)$ is given by

$$
\left\{\begin{array}{l}
G(t, \tau)=\frac{1}{6} \tau^{2}(3 t-\tau), \quad 0 \leqslant \tau \leqslant t \leqslant 1 \\
t^{2}(3 \tau-t), \quad 0 \leqslant t \leqslant \tau \leqslant 1
\end{array}\right.
$$

and

$$
0 \leqslant G(t, \tau) \leqslant \frac{1}{2} t^{2} \tau, \quad \forall t, \tau \in[0,1]
$$


Let us review the mathematical background (see [10], [16]). Let $X=$ $=C([0,1], \mathbb{R})$ be the set of all non-negative real-valued continuous functions on the interval $[0,1]$, and let this set be endowed with the norm $\|x\|=\|x\|_{\infty}+\left\|x^{\prime}\right\|_{\infty}$, where $\|x\|_{\infty}=\sup _{t \in[0,1]}|x(t)|$. Also, let $E=$ $=C^{1}([0,1], \mathbb{R})$ and $P=\{x \in E: x(t) \geqslant 0$ for all $t \in[0,1]\}$. Define a mapping $d: X \times X \rightarrow E$ by $d(x, y)=e^{v} \sup _{t \in[0,1]}|x(t)-y(t)|$ for all $x, y \in X$ and $v \in[0,1]$. Also, consider the partial order $x \sqsubseteq y$ iff $x(t) \leqslant y(t)$ for all $t \in[0,1]$. Evidently, $(X, \sqsubseteq)$ is a partially ordered set and $(X, d)$ is a complete cone metric space. Further, consider the $c$-distance $q: X \times X \rightarrow E$ given by $q(x, y)=e^{v} \sup _{t \in[0,1]}|y(t)|$ for all $x, y \in X$ and $v \in[0,1]$. Moreover, we use the following assumptions:

(I) There exists $\alpha: X \rightarrow[0,1)$ such that

$$
0 \leqslant k(t, y(t)) \leqslant 4 \alpha(x) q(x, y) e^{-v}
$$

for all $x, y \in X$ with $x \sqsubseteq y$ and for all $t \in[0,1]$ and

$$
\alpha\left(\int_{0}^{1} G(t, \tau) k(\tau, x(\tau)) d \tau\right) \leqslant \alpha(x) \forall x \in X .
$$

(II) There exists $x_{0} \in X$ such that $x_{0}(t) \leqslant \int_{0}^{1} G(t, \tau) k\left(\tau, x_{0}(\tau)\right) d \tau$ for all $t \in[0,1]$; so, the integral equation (11) admits a lower solution in $X$. Suppose that the function $k \in C([0,1] \times \mathbb{R}, \mathbb{R})$ satisfies assumptions (I) and (II). Now, we prove the existence of at least one solution of (10) in $X$. This problem is equivalent to the fixed-point problem obtained by introducing the continuous integral operator $T: X \rightarrow X$ given as

$$
(T x)(t)=\int_{0}^{1} G(t, \tau) k(\tau, x(\tau)) d \tau, \quad t \in[0,1], \quad x \in X .
$$

Now, we prove that the operator $T$ satisfies all the conditions in Corollary 1. Consequently, there exists a fixed point of $T$ in $X$. Since $k \in C([0,1] \times \mathbb{R}, \mathbb{R})$ is nondecreasing, we conclude that $T$ is a nondecreasing mapping with respect to $\sqsubseteq$. Also, by using (12) and (13), we obtain

$$
q(T x, T y)=e^{v} \sup _{t \in[0,1]} \int_{0}^{1} G(t, \operatorname{tau}) k(\tau, y(\tau)) d \tau \leqslant \alpha(x) q(x, y)
$$

for all $t \in[0,1]$ and for all $x, y \in X$ with $x \sqsubseteq y$. By assumption (II), there exists $x_{0} \in X$ such that $x_{0} \sqsubseteq T x_{0}$. Also, from (14) and since $\alpha$ assumes 
values in the interval $[0,1)$, we have $\alpha(T x) \leqslant \alpha(x)<1$ for all $x \in X$. Thus, all the conditions of Corollary 2 with $\nu_{1}(x)=\alpha(x)$ and $\nu_{i}(x)=0$ for $i=2,3,4,5$ hold true. Hence, we deduce the existence of a fixed point of $T$; that is, there exists a solution of problem (10) in $X$.

4. Conclusion. In this paper, we have considered the condition of orbitally $G$-continuity of mappings instead of the condition of continuity of mappings and cone metric spaces endowed with graph instead of cone metric spaces; some theorems of existing literature, such as Kada et al. [11], Cho et al. [3], Fallahi et al. [4], Fallahi and Soleimani Rad [5], Petrusel and Rus [13], Rahimi and Soleimani Rad [15], [16], and Wang and Guo [17] can be unified there. We finish this paper with some questions:

Question 1. Can one obtain these results by considering nother condition instead of continuity of the mapping $T$ ?

Question 2. Can one prove the main theorem and its corollaries by considering one contractive-type relation instead of two contractive-type relations?

Acknowledgment. The authors thank the Editorial Board and the referees for their valuable comments that helped to improve the text.

\section{References}

[1] Alfuraidan M. R., Khamsi M. A. Coupled fixed points of monotone mappings in a metric space with a graph, Fixed Point Theory., 2018, vol. 19, no. $1,33-44$.

[2] Bondy J. A., Murty U. S. R., Graph theory, Springer, 2008.

[3] Cho Y. J., Saadati R., Wang S. H. Common fixed point theorems on generalized distance in ordered cone metric spaces, Comput. Math. Appl., 2011, vol. 61, pp. 1254-1260.

[4] Fallahi F., Petrusel A., Soleimani Rad G. Fixed point results for pointwise Chatterjea type mappings with respect to a c-distance in cone metric spaces endowed with a graph, U.P.B. Sci. Bull. (Series A)., 2018, vol. 80, no. 1, pp. $47-54$.

[5] Fallahi K., Soleimani Rad G. Fixed points of a $\varphi$-G-contractive mapping with respect to a c-distance on an abstract metric space endowed with a graph, Mathematical Notes., 2019, vol. 105, no. (5-6), pp. 781-788.

[6] Hardy G. H., Rogers T. D. A generalization of a fixed point theorem of Reich, Canad. Math. Bull., 1973, vol. 16, pp. 201-206.

[7] Huang H., Radenović S., Dosenović T. Some common fixed point theorems on c-distance in cone metric spaces over Banach algebras, Appl. Comput. Math., 2015, vol 14, no. 2, pp. 180-193. 
[8] Huang L. G., Zhang X. Cone metric spaces and fixed point theorems of contractive mappings, J. Math. Anal. Appl., 2007, vol. 332, pp. 1467-1475.

[9] Jachymski J. The contraction principle for mappings on a metric space with a graph, Proc. Amer. Math. Soc., 2008, vol. 136, pp. 1359-1373.

[10] Jleli M., Čojbas̆ić Rajić V., Samet B., Vetro C. Fixed point theorems on ordered metric spaces and applications to nonlinear elastic beam equations, J. Fixed Point Theory Appl., 2012, vol. 12, pp. 175-192.

[11] Kada O., Suzuki T., Takahashi W. Nonconvex minimization theorems and fixed point theorems in complete metric spaces, Math. Japon., 1996, vol. 44, pp. 381-391.

[12] Nicolae A., O'Regan D., Petrusel A. Fixed point theorems for singlevalued and multivalued generalized contractions in metric spaces endowed with a graph, Georgian Math. J., 2011, vol. 18, pp. 307-327.

[13] Petrusel A., Rus I. A. Fixed point theorems in ordered L-spaces, Proc. Amer. Math. Soc., 2006, vol. 134, no. 2, pp. 411-418.

[14] Petrusel A., Rus I. A. Fixed point theory in terms of a metric and of an order relation, Fixed Point Theory., 2019, vol. 20, no. 2, pp. 601-622.

[15] Rahimi H., Soleimani Rad G. Common fixed-point theorems and c-distance in ordered cone metric spaces, Ukrain. Math. J., 2014, vol. 65, no. 12, pp. $1845-1861$.

[16] Soleimani Rad G., Rahimi H., Vetro C. Fixed point results under generalized c-distance with application to nonlinear fourth-order differential equation, Fixed Point Theory., 2019, vol. 20, no. 2, pp. 635-648.

[17] Wang S., Guo B. Distance in cone metric spaces and common fixed point theorems, Appl. Math. Lett., 2011, vol. 24, pp. 1735-1739.

[18] Zabrejko P. P. K-metric and K-normed linear spaces: survey, Collect. Math., 1997, vol. 48, pp. 825-859.

Received July 20, 2019.

In revised form, February 4, 2020.

Accepted February 7, 2020.

Published online February 18, 2020.

Department of Mathematics, Faculty of Science

Central Tehran Branch, Islamic Azad University, Tehran, Iran

E-mail: ladan.aryan759@gmail.com; rahimi@iauctb.ac.ir;

gha.soleimani.sci@iauctb.ac.ir 